Jeu

Revue de théâtre

\title{
Les attracteurs étranges
}

\section{Brunella Eruli}

Numéro 55, juin 1990

URI : https://id.erudit.org/iderudit/26960ac

Aller au sommaire du numéro

Éditeur(s)

Cahiers de théâtre Jeu inc.

ISSN

0382-0335 (imprimé)

1923-2578 (numérique)

Découvrir la revue

Citer cet article

Eruli, B. (1990). Les attracteurs étranges. Jeu, (55), 28-34.

Ce document est protégé par la loi sur le droit d'auteur. L'utilisation des services d'Érudit (y compris la reproduction) est assujettie à sa politique d'utilisation que vous pouvez consulter en ligne.

https://apropos.erudit.org/fr/usagers/politique-dutilisation/
Cet article est diffusé et préservé par Érudit.

Érudit est un consortium interuniversitaire sans but lucratif composé de l’Université de Montréal, l'Université Laval et l'Université du Québec à Montréal. Il a pour mission la promotion et la valorisation de la recherche. https://www.erudit.org/fr/ 

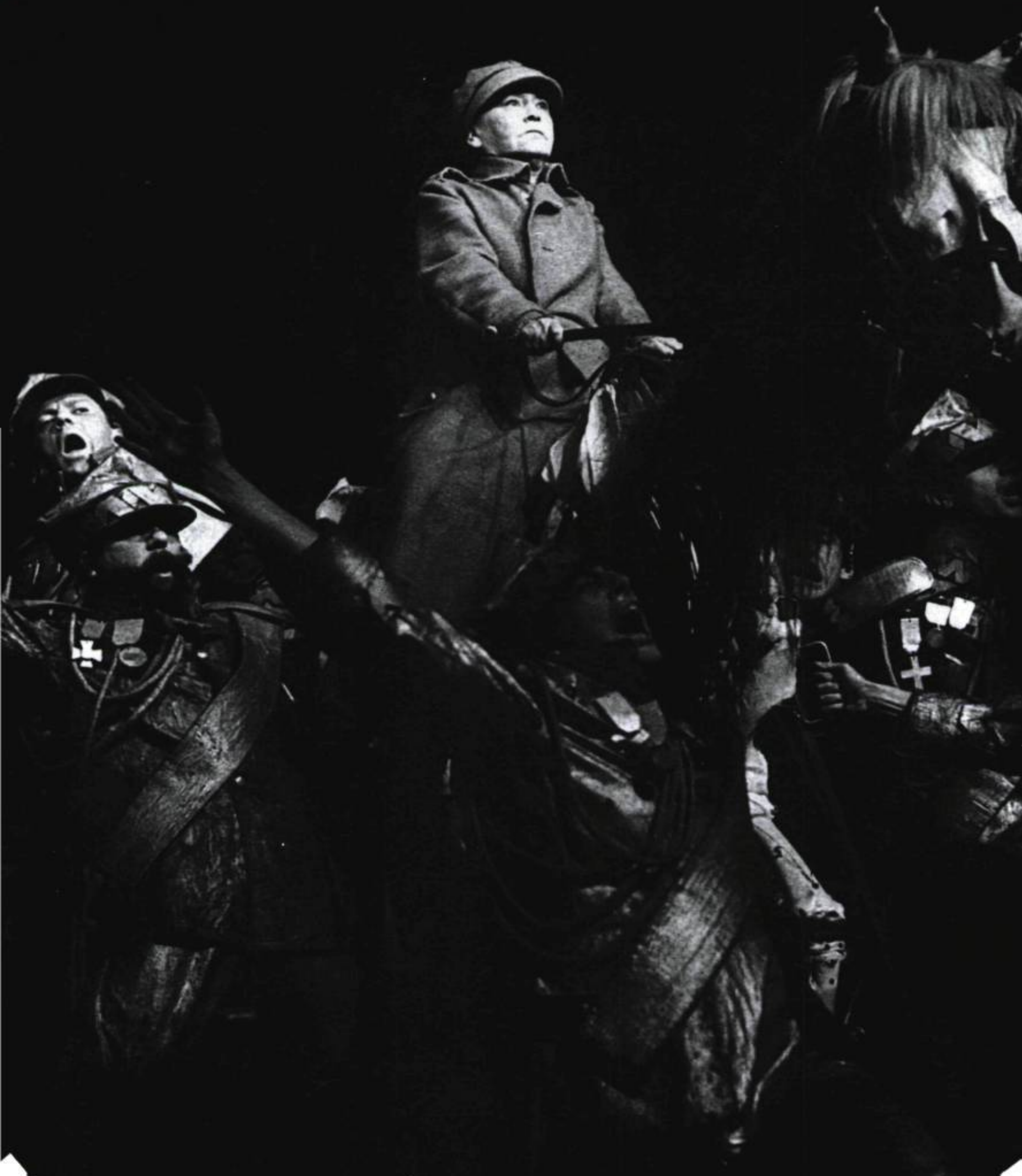


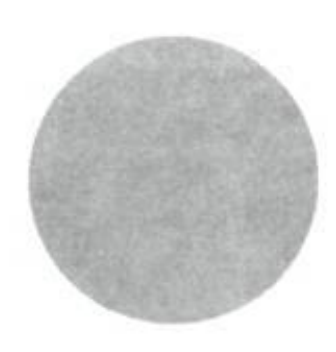

\section{les attracteurs étranges}

Quel que soit l'impact du langage fractal du côté des poètes (artistes ou mathématiciens), et quel que soit l'intérêt de ses racines historiques, le test le plus ardu et sans doute le plus important de ce langage se trouve ailleurs. Ce langage est-il utilisable et flexible au niveau de la prose la plus utilitaire? Est-il utilisable et flexible, non pas demain mais aujourd'hui, au niveau de la prose la plus raffinée?

Aux deux questions [...] il y a des réponses affirmatives. [...] Ma foi dans les vertus de l'explicarion est tellement inébranlable, qu'elle n'a aucune peine à se passer de gestes de dévotion; elle est si exigeante qu'elle n'aime invoquer les grands principes que quand il $y$ a lieu de le faire.

"La prose fractale devant la confrontation entre l'homme d'action et la philosophie", in Mandelbrot, les Objets fractals, Paris, 1989, p. 211

Je reviens une fois encore, une fois de plus, vers ce lieu de l'âme qui, depuis longtemps, provoque ma réflexion sur le sens des choses, des images qu'on se fait d'elles. C'est là que j'ai accepté ma finitude ou que je l'ai reconnue, faute de pouvoir vraiment l'assumer. C'est là que j'ai nourri ma réflexion sur cet événement extraordinaire qu'est la naissance d'une cuvre d'art. Et pourtant, je crois pouvoir dire non pas - comme Kantor — que je n'y reviendrai jamais, mais que peut-être je ne suis jamais parvenue à l'atteindre. Ce qui m'intéresse dans le théâtre de Kantor, c'est ce que je ne parviens pas encore à voir, ce à quoi je suis aveugle. Ma cécité, si elle provoque un sentiment de perte, nourrit ma conscience. Avouer cela, ce n'est pas toujours facile. Le cheminement vers quelque chose qui échappe lorsqu'on croyait l'avoir cerné est un enjeu de toute activité critique et le sujet des propos que je voudrais faire partager. L'image que nous avons d'un spectacle théâtral, dans l'instant et la durée, est le résultat d'une rencontre entre plusieurs variables subjectives, d'ordre psychologique, mais aussi culturel et historique. L'évolution d'un de ces éléments modifie cette image d'une manière sensible, mais elle est difficile à cerner, à quantifier et encore plus à décrire. Le critique se trouve confronté non pas à une image fixe, toujours égale à elle-même et donc connaissable, mais à des formes semblables à celles qu'en géométrie fractale on appelle *attracteurs étranges", donc à

- Le thème de la prison. Kantor l'applique a l'cuvre d'art dans un passage de Qu ils crituent lo artistes' II suggère par là que la mémoire et la création sont en quelque sorte des prisons.- Photo: Jacquie Bablet/CNRS. des significations, à des images qui ne sont jamais parfaitement identiques, ni prévisibles. Ce chaos régi par des lois - instables - fait surgir une tension, un sentiment d'inquiétude auxquels la réflexion critique tâche de donner une réponse.

Qu'est-ce que l'on voit lorsqu'on regarde? Comment "re-voir" ce que l'on connait déjà ou que l'on croit connaître? Stendhal faisait déjà la distinction entre le souvenir continu, organisé, donc artificiel et fabriqué, et le souvenir mouvant, à la limite indicible, tel un fragment des fresques du Camposanto 


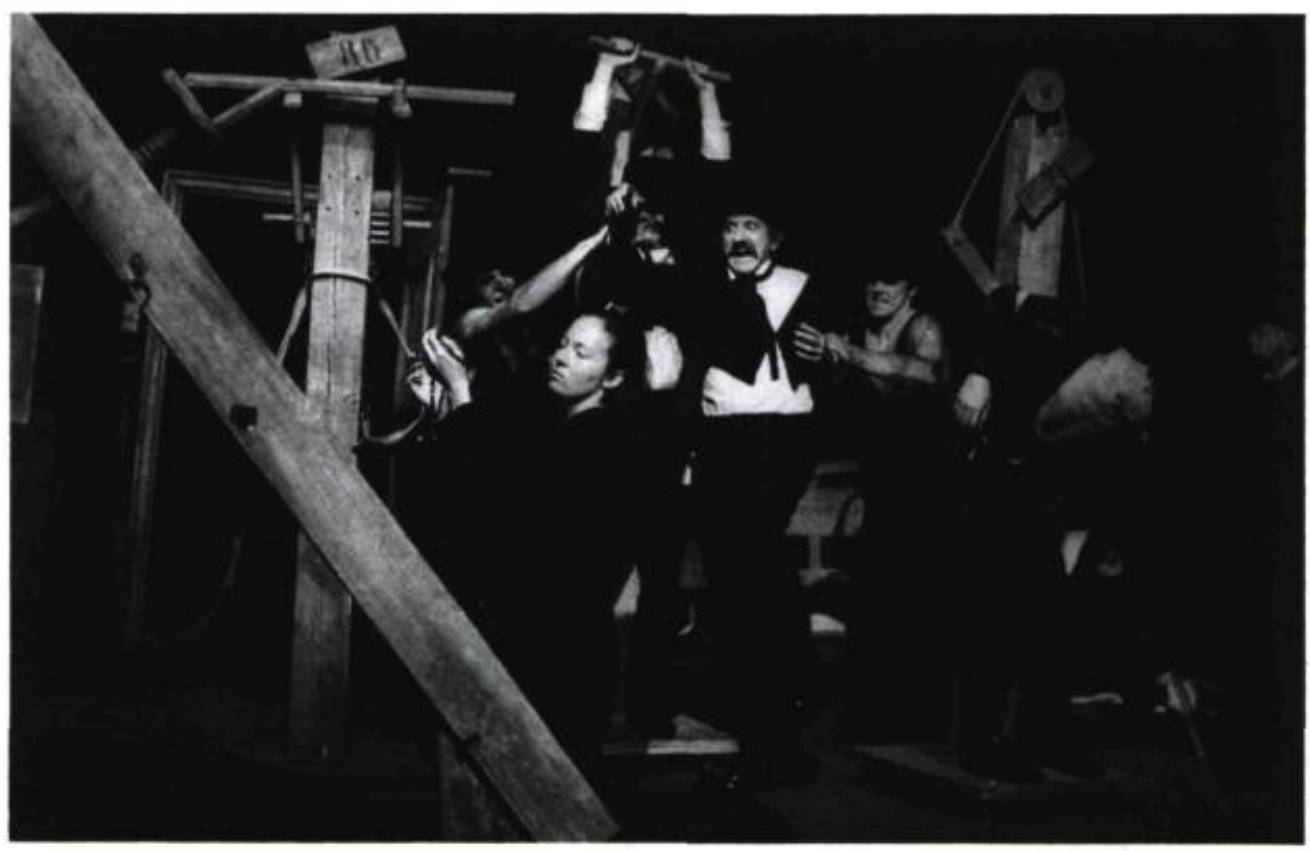

Quils crivent les artistes! *Kantor a fair de la mémoire une ligne de force de sa création. [...] Les ruines sont aussi difficiles d assembler qu'a détruire entièrement. Photo : Jacquie Bablet/ CNRS.

de Pise dont de larges pans seraient tombés. Par le fait même de "transcoder" le spectacle ou un événement visuel, de le «reverser» dans le langage, le critique fige la matière mouvante de la réalité artistique. Il peut alors déclarer que le spectacle - désormais terra cognita - "est" ceci ou cela, qu'il est, en somme, un objet analysable. À partir de ce moment, le souvenir qu'il en garde se cristallise et, entre le spectateur et le spectacle qu'il revoit, il devient comme un écran, tissé par des images déjà structurées, devenues familières et aussi plus facilement repérables ou "transcodables".

C'est à travers mon expérience du théâtre de Kantor que j'ai compris le danger caché dans le regard de Méduse que le critique peut projeter sur le spectacle. On dirait que ce regard, s'il fige l'objet regardé, peut aussi figer celui qui le porte.

Le spectateur peut-il devenir "prisonnier" de sa propre mémoire? Le thème de la prison, Kantor l'applique à l'œuvre d'art dans un passage de Qu'ils crèvent les artistes! Il suggère par là que la mémoire et la création sont en quelque sorte des prisons. L'artiste et le spectateur seraient-ils, l'un comme l'autre, prisonniers aussi de leur mémoire?

Revoir ce que l'on connaît déjà ou que l'on croit connaître provoque souvent une gêne. À côté de la terra cognita, on perçoit des images et des significations nouvelles qui nous laissent incertains sur nos propres impressions. Vont-elles les confirmer ou non? Sont-elles un trouble passager de la vision? Faute d'avoir été reconnues dans leur nouveauté, ces perceptions sont absorbées dans le moule préexistant. À ce que nous avions ressenti et compris jadis, nous restons attachés par une sorte de paresse, en réalité par la crainte de nous aventurer sur un terrain inconnu. Dans cette incertitude s'enracine l'attente de saisir ce que nous savons avoir jusque-là manqué. Un désir - peut-on dire la nostalgie? - d'atteindre une perception globale se fait jour. Est-ce là une manifestation de l'attesa molto importantel dont parle Kantor?

1. L'attente très importante. N.d.I.r. 
-Kantor applique le paradoxe de Diderot sur le comédien : Kantor joue ici son propre rôle comme l'acteur qui, sur scène. joue le rôle de l'acteur. II déréalise ainsi sa présence. sa personne ici et maintenant, il fait de luimème, de sa propre vie. un objet figet, une citation." Je ne neviendrai jamais. Photo : Jacquie Bablet/CNRS.
Kantor a fait de la mémoire une ligne de force de sa création. Il n'est pas le seul, mais son apport demeure original par le caractère obsessionnel, chez lui, de ce procédé. Au fil des spectacles, d'une façon de plus en plus consciemment assumée et soulignée, il reprend les éléments épars — choisis par sa mémoire ou par son regard de peintre, peu importe - pour révéler non leur continuité éventuelle, mais leur discontinuité irrévocable. Dans cette perspective, la mémoire ne retrouve pas le temps perdu, elle ne complète pas non plus l'objet parcellisé. Les ruines sont aussi difficiles à assembler qu'à détruire entièrement. On repense alors à $U b u$ esclave qui déclarait : "On n'aura pas tout détruit tant qu'on n'aura pas détruit même les ruines."

Les mêmes situations inachevées, les mêmes fragments reviennent mais jamais à l'identique. Elles font naître ainsi, chaque fois, l'espoir d'un changement possible, d'une épiphanie qui enfin s'accomplirait, mais celle-ci retombe et se perd comme une détumescence inexplicable.

Les images de Kantor évoquent des images fractales: Kantor segmente la réalité qu'il fige en des clichés-souvenirs. Ces fragments, il les assemble dans le cadre privilégié de la scène ou du tableau, mais l'image obtenue repropose le même sentiment de fragmentation et de perte de la totalité.

Le système construit par Kantor est basé sur un antagonisme entre deux polarités qui contiennent à la fois l'espoir du changement et l'évidence de son impossibilité: l'essentiel est opposé au quotidien, les vivants servent à émouvoir les morts, ou vice versa, le blasphématoire veut évoquer le sacré, la mémoire envahit le présent. Dans le champ ainsi limité, ces forces antagonistes aboutissent à la création de lignes qui ne peuvent que dessiner des formes quasi identiques, et pourtant jamais parfaitement prévisibles dans toutes leurs composantes. Le hasard et le déterminisme se disputent le terrain, ou plutôt ils se relaient, car dans certains systèmes chaotiques, le hasard est strictement déterminé.

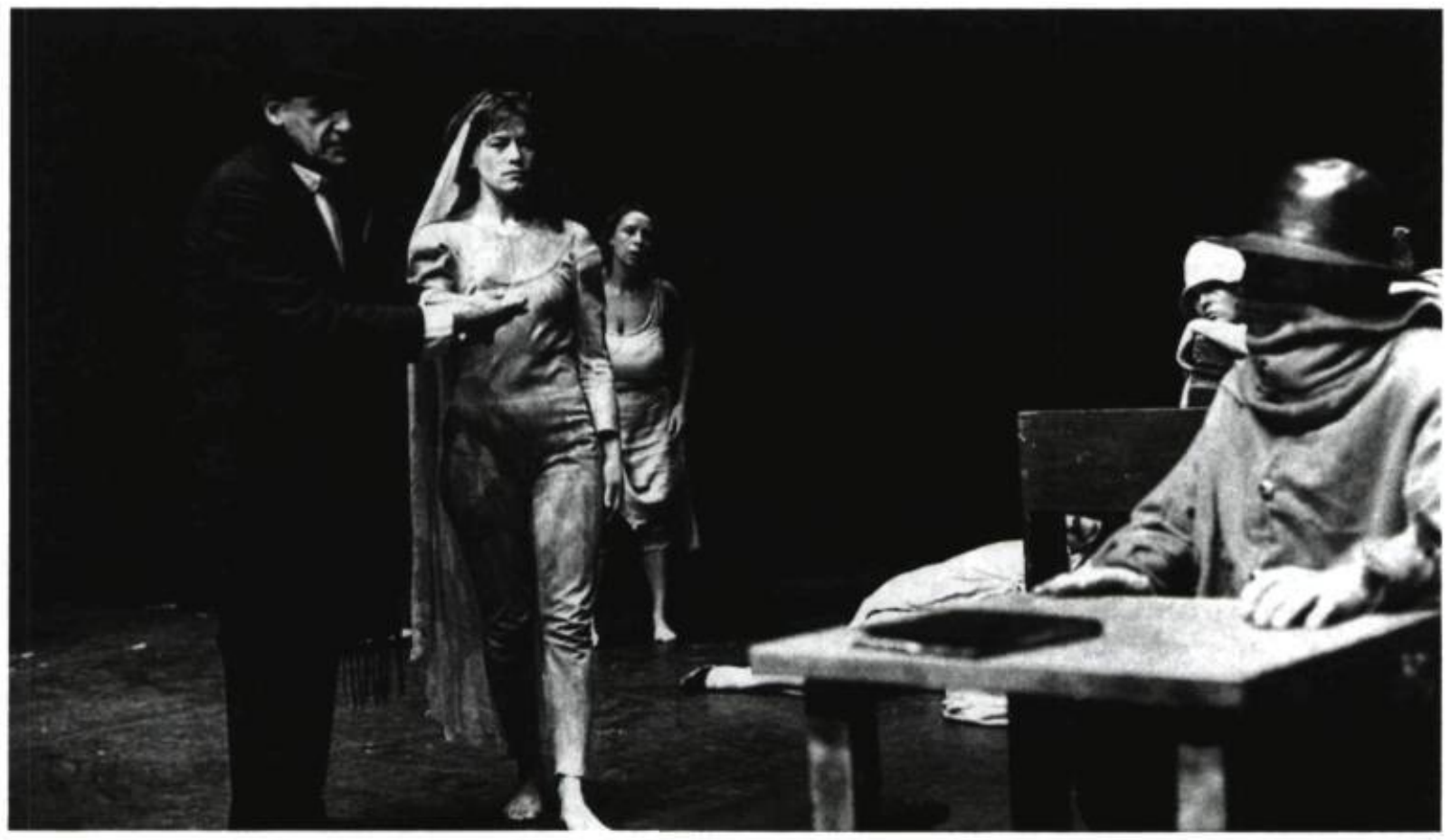


Une partie importante de l'œuvre de Kantor se place ainsi sous le signe d'un mécanisme qui pourrait rappeler la compulsion de répétition. Ce qui demeure incompris fait retour, s'actualisant sur la scène. Telle une âme en peine à la recherche de sa délivrance, Kantor explore son passé historique, personnel, affectif. Cette recherche est le moteur d'un mécanisme de répétition qui se nourrit de lui-même, mais son dépassement se réalise grâce à l'exaspération volontaire, acharnée, de ce mécanisme même. La répétition devient alors la seule réalité connaissable et concrètement expérimentée.

Volontairement recherchée, la répétition crée une grammaire, un langage qui déjoue les capacités métaphoriques du théâtre, car le référent veut se donner uniquement par rapport à lui-même. En fait la seule réalité des choses repose sur une présence théâtrale, à l'intérieur d'un système construit par Kantor avec une obstination véritablement hérö̈que. Une chaise de Kantor n'est jamais - mais l'a-t-elle jamais été? - une simple chaise du premier degré de la réalité. La chaise devient un objet déréalisé, comme un mot qui aurait perdu sa première signification pour n'exister que dans des expressions toutes faites que l'on emploie sans plus se rendre compte de leur signification d'origine. Ce n'est pas la réalité qui a effacé le théâtre, mais le théâtre qui l'a déréalisée.

Le retour au théâtre en tant que représentation (de quoi, au juste?) et non en tant qu'événement, est particulièrement clair dans le dernier spectacle de Kantor, Je ne reviendrai jamais. Ici, dans sa pratique, Kantor renverse sa théorie sur les rapports entre la réalité et l'illusion. La tension qui polarise ces deux éléments bascule : on a l'impression que tout est seulement illusion, représentation sans autre référent que théâtral. Ce vide, maintenant, est pleinement assumé.

On dirait même que, dans ce spectacle, Kantor applique le paradoxe de Diderot sur le comédien : Kantor joue ici son propre rôle comme l'acteur qui, sur scène, joue le rôle de l'acteur. Il déréalise ainsi sa présence, sa personne ici et maintenant, il fait de lui-même, de sa propre vie, un objet figé, une citation. Sur scène, il accomplit toujours les mêmes gestes, sa voix, enregistrée, ne pourra répéter que les mêmes phrases. L'organisation de l'espace trahit ce virage : les portes de Je ne reviendrai jamais sont de véritables portes de théâtre, elles ne servent qu'à faire entrer et sortir, d'une manière prévisible, les personnages et Kantor lui-même.

L'utilisation référentielle de la mémoire exacerbe le sentiment d'incomplétude lié au souvenir, mais déclare aussi l'impossibilité de vivre le présent. La seule réalité qui peut fonctionner comme pierre de touche pour et contre la mémoire, afin qu'elle devienne davantage explicite ou bien afin qu'elle s'efface pour permettre d'observer les événements à neuf, c'est Kantor lui-même. Rechercher la certitude de la réalité sur une scène de théâtre, c'est se vouer à la déception. Du coup, alors, ce retour à une théâtralisation affichée serait-il une déclaration implicite que la réalité est inconnaissable, qu'on ne peut pas percer la surface des apparences et que, comme il le dit, plus loin... rien...?

Après avoir construit des cercles concentriques de plus en plus inexorables, Kantor s'est enfermé dans l'espace d'une mémoire qui est peut-être la sienne, mais qui est exclusivement théâtrale et théâtralisée. On a l'impression que sa propre histoire, sa propre vie existent et prennent sens uniquement dans la mesure où elles prennent forme sur la scène et grâce à elle. Cette constatation, encore plus radicale et désespérée que la réalité est perdue, oubliée, jette une lumière nouvelle aussi sur l'œuvre précédente de Kantor.

Regardant les quatre spectacles ${ }^{2}$ d'affilée, on retrouve de l'un à l'autre des répétitions obsédantes, des trajectoires identiques, mais il y a aussi des changements importants. Les spectacles déteignent les 


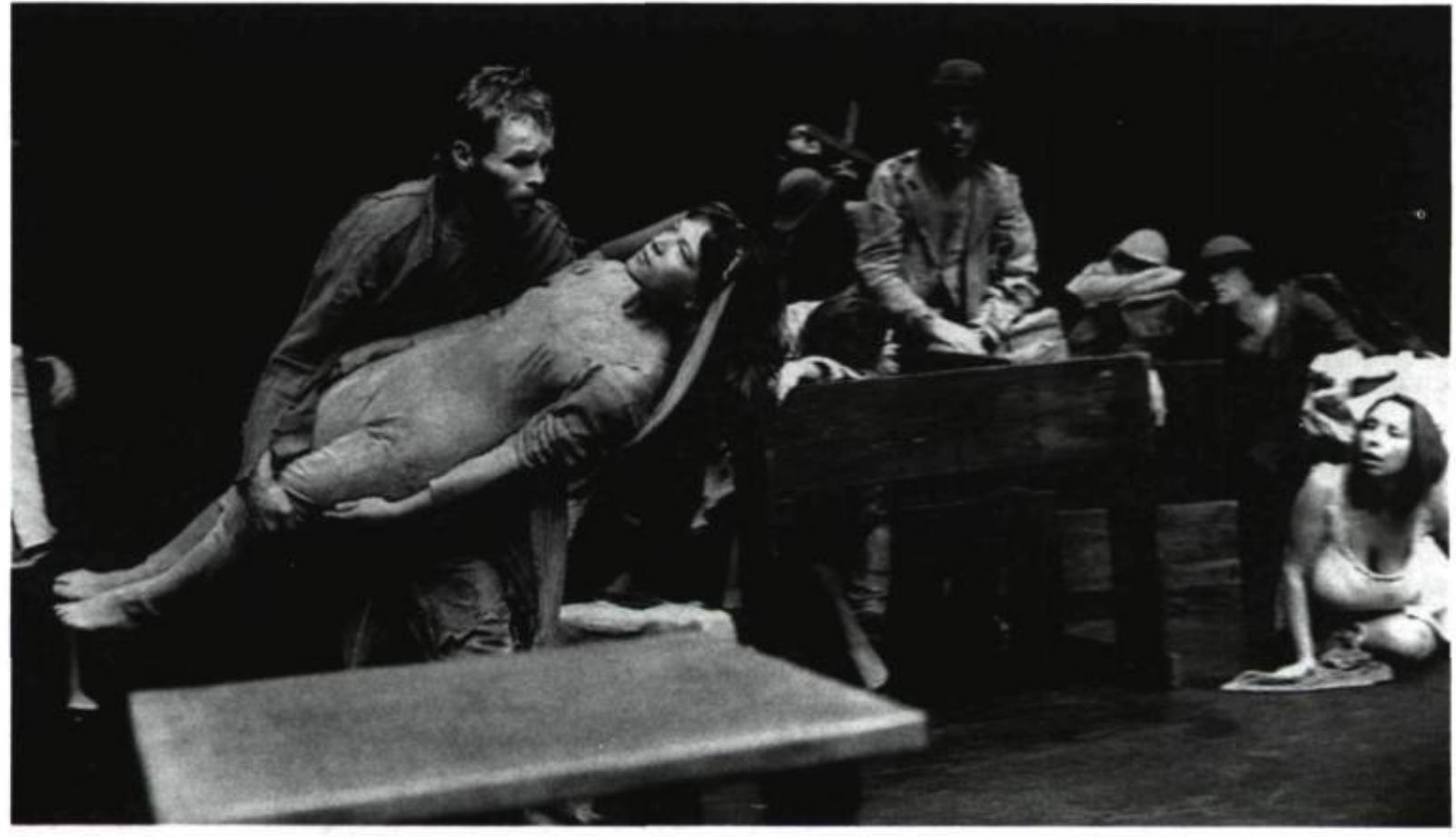

Je ne reviendrai jamatis. Photo : Jacquic Bablet/ CNRS.

- Les spectacles déteignent les uns sur les autres, on retrouve des rimes, des chiasmes, des rappels volontaires et

involontaires (comme on peut parler d'une mémoire volontaire et d'une mémoire involontaire) bref, une rhétorique de l'image et du sens.-

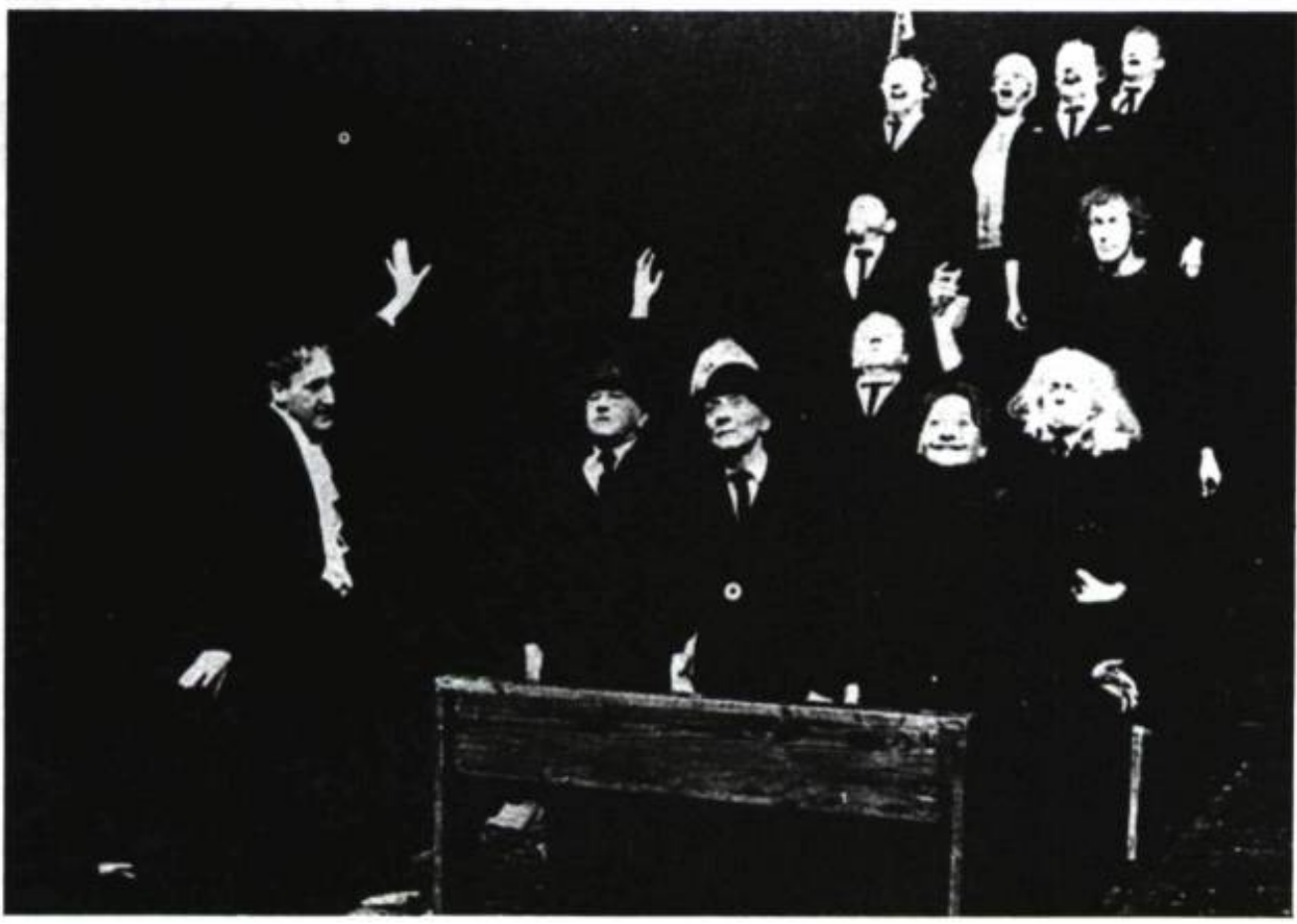


uns sur les autres, on retrouve des rimes, des chiasmes, des rappels volontaires et involontaires (comme l'on peut parler d'une mémoire volontaire et d'une mémoire involontaire) bref, une rhétorique de l'image et du sens. Cette rhétorique, qui a toujours été présente, montre de plus en plus ses mécanismes, ses matériaux, épurés par un long séjour dans les limbes de la représentation, pour mieux faire ressortir le caractère dérisoire de toute entreprise de représenter une réalité, ce qui ne peut rester qu'un souhait. Nostalgie de la terre où l'on ne reviendra jamais, mais y a-t-on jamais véritablement vécu?

L'obsession "citadelle" de ce spectacle, son côté autocannibale, l'autocombustion qui engendre et entretient le pari de Je ne reviendrai jamais a quelque chose d'héroïque et d'étouffant. La cruauté de cet engrenage, qui finit par séquestrer Kantor, est révélée par l'étrange manque d'humour de cette pièce, toute placée sous la couleur du grotesque. Il est toutefois clair que Kantor, en réalité, est ici en train de jouer une partie avec lui-même et qu'il joue à la fois le rôle du Commandeur et celui de Dom Juan. Au spectateur est attribué le rôle de Sganarelle, il ne pourra suivre son maître en enfer, mais il ne se placera pas non plus du côté du Commandeur et, en plus, il se sentira trahi par son maître.

Le retour dont parle Kantor est-il un vrai retour, un retour à soi qui présuppose un changement, une évolution, ou bien s'agit-il du retour à un point d'une spirale sans fin, comme les "anneaux étranges" dont Hofstadter parle au sujet du canon de l'Offrande musicale de Bach?

Jusqu'à quel point fonctionnent les attracteurs étranges au théâtre? Peut-on sortir d'une réalité perçue comme dégradée et dépourvue de sens sans pour autant s'enfermer dans une réalité qui n'est que représentation (à l'identique)? Le désespoir de ce dernier titre, loin d'annoncer une renonciation, ne pourrait-il annoncer un changement? Picasso ne disait-il pas que le style, c'est quand on est mort?

\section{brunella eruli}

-L'obsession ecitadeller de ce spectacle, son côté autocannibale,

Tautocombustion qui engendre et entretient le pari de Je ne reviendrai jamais a quelque chose d'héroique et d'étouffant.: Photo: Jacquic Bablet CNRS.

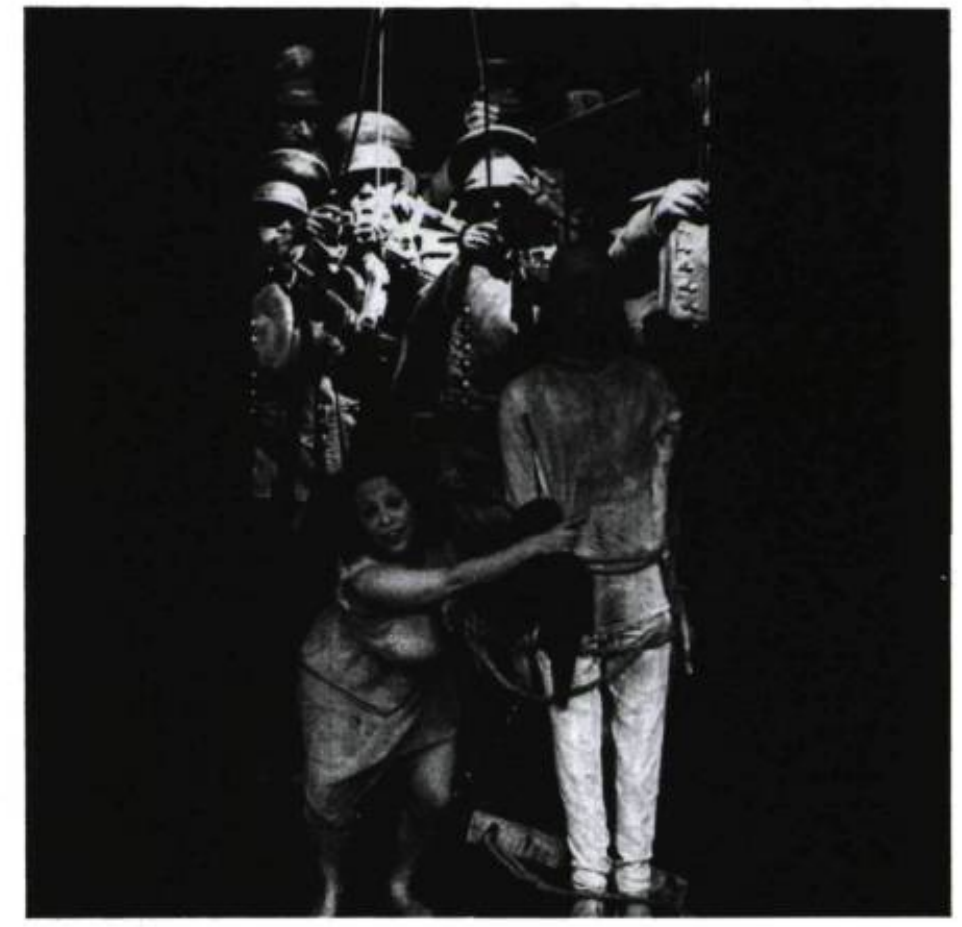

